\title{
Attitude Determination from Single Camera Vector Observations
}

\author{
Vasko Sazdovski, Peter M G Silson, Antonios Tsourdos \\ Autonomous Systems Group, Department of Informatics and Sensors \\ Defence Academy of the United Kingdom, Cranfield University \\ Shrivenham, Swindon SN6 8LA, United Kingdom \\ v.sazdovski@cranfield.ac.uk, p.m.g.silson@cranfield.ac.uk, a.tsourdos@cranfield.ac.uk
}

\begin{abstract}
Attitude determination is of major importance in Guidance and Control Systems of the Unmanned Aerial Vehicles (UAV's). Supplying wrong or imprecise attitude can very often be catastrophic for the UAV's. Vision sensors are nowadays essential as they provide a rich source of information given as relative measurements between the vehicle navigation parameters (position, velocity and attitude) and the environment. This paper presents a framework for attitude determination from single camera vector observations. We assume a known environment in a form of a map and true vehicle positions from which each observation has been taken.

Two different methods for attitude determination are presented: an iterative numerical solution based on Gauss Newton's method and an exact method known as the Davenport q-method. Pros and cons of the both solutions are presented.
\end{abstract}

Keywords- attitide; quaternions; single camera; vector observations; Gauss Newton's method; Davenport q-method.

\section{INTRODUCTION}

Today UAV's have become an indispensable ingredient for many applications where human operation is considered unnecessary, too dangerous or impossible [1]. These applications include planetary explorations, environmental and climate research and monitoring, traffic monitoring, inspection of man-made structures such as power lines and pipelines, urban planning, pollution studies and many others. Many of today's intelligence gathering, surveillance and reconnaissance missions cannot be carried out without UAV's.

All these applications and missions require UAV's to operate in a partially known or known environment, where we have some priori knowledge of the environment.

Typically in these applications and missions the need for safety arises. Safety here simply implies that the UAV's are not damaged or destroyed during the mission [2]. Apart from factors such as vehicle constraints, environment factors (strong winds, icing, thunderstorms) and enemy fire, reliable navigation parameters play a crucial role in the safety of the UAV's. Supplying wrong or imprecise navigation parameters (position, velocity and attitude) to the Guidance and Control System can, very often, be catastrophic for the UAV's.
In general the attitude of a rigid body in space is a representation of the instantaneous orientation of one coordinate frame (body-fixed coordinate frame) to a second coordinate frame (inertial coordinate frame). The axes of the body and inertial frames are related by a linear transformation commonly given by an orthogonal matrix usually referred to as matrix of transformation i.e. transformation matrix [3]. This matrix may be represented by various sets of coordinates: Euler angles, quaternions, etc. The quaternion approach is often the preferred implementation as the linearity of the quaternions, the lack of trigonometric functions, and the small numbers of parameters that allow efficient implementation. Quaternion parametrizations are singularity free and are more computationally efficient then the Euler angles [4]. The only shortcoming of the quaternions is that they don't have simple geometrical representation and because of this can't be measured directly.

Vision sensors are essential nowadays, primarily because they are cost effective, small, compact and reliable passive sensors and secondarily are capable of providing a rich source of information about the vehicle environment.

A single camera is the desired hardware setup for UAV's, it provides a rich source of information given as relative measurements between the vehicle navigation parameters (position, velocity and attitude) and the environment. Because of their nature of being relative they interconnect the navigation parameters and the environment. If we have known environment using the relative measurements we can determine the vehicle navigation parameters and vice versa, if we have perfect vehicle navigation parameters we can create a map of the environment. The major drawback of the single camera is the absence of the depth information i.e. relative range to the map points.

In this paper we assume known environment in a form of a map (set of known map points) and true vehicle positions from where the single camera vector observations has been taken. Both the map points and the vehicle positions are given in the navigation frame which represents an inertial frame. Here we give proper framework for attitude determination from single camera vector observations. We show that the single camera vector observation of a map point and its replica vector 
formed from the vehicle position and the map point are sufficient for determination of the vehicle attitude.

We use two different methods for determination of the solution. The first method is an iterative numerical solution based on Gauss Newton's method and the second is the more elegant closed form solution known as the Davenport q-method, solution to the Wahba's problem.

Section 2 describes the single camera setup on the vehicle. The camera vector observations presented as vector application are described here. Section 3 and 4 are devoted to the Gauss Newton's method and Davenport q-method respectively. The simulation results are presented in section 5, where the pros and cons of the both methods are elaborated. The conclusions and the future work are part of section 6 .

\section{SINGLE CAMERA SETUP}

We assume that the single camera is mounted on such a way that the camera frame $\left(P ; x_{c}, y_{c}, z_{c}\right)$ is at the center of gravity of the vehicle or very near to the center of gravity and is aligned with the vehicle body frame. The origin of the camera frame is at the perspective center $P$ of the camera i.e. the center of gravity of the vehicle.

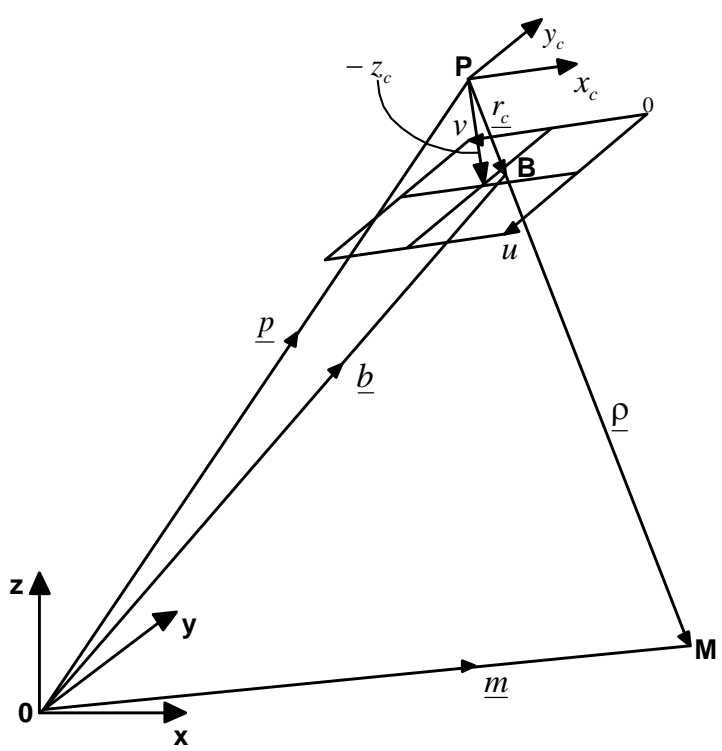

Figure 1. Single camera setup (vectors application)

The $x_{c}$ axis is defined in forward direction and is perpendicular to the horizontal component $u$ of the camera image. The $z_{c}$ axis is perpendicular to $x_{c}$ axis and is equal to the focal length of the camera.

The negative sign appears when $z_{c}$ axis is pointing to the center of the camera image. The $y_{c}$ axis completes the right handed orthogonal coordinate system and is perpendicular to the vertical component $v$ of the camera image, see figure 1 .
IEEE Intelligent Systems Conference, London, July 2010

In the camera frame we measure the camera vector $\underline{r}_{c}$. The unit vector in the direction $\underline{r}_{c}$ usually notated as $\underline{\hat{r}}_{c}$ may be evaluated as

$$
\hat{\underline{r}}_{c}=\frac{\underline{\boldsymbol{r}}_{c}}{\left|\underline{\boldsymbol{r}}_{c}\right|} .
$$

Vector $\underline{r}_{c}$ can be transformed in the navigation frame with $C_{b 2 n}$, the transformation matrix that transforms body frame coordinates into navigation frame coordinates:

$$
\underline{r}=C_{b 2 n} \underline{r}_{c}
$$

where $C_{b 2 n}$ given in quaternion form is [4]:

$$
C_{b 2 n}=\left[\begin{array}{ccc}
\left(q_{0}^{2}+q_{1}^{2}-q_{2}^{2}-q_{3}^{2}\right) & 2\left(q_{1} q_{2}-q_{0} q_{3}\right) & 2\left(q_{1} q_{3}+q_{0} q_{2}\right) \\
2\left(q_{1} q_{2}+q_{0} q_{3}\right) & \left(q_{0}^{2}-q_{1}^{2}+q_{2}^{2}-q_{3}^{2}\right) & 2\left(q_{2} q_{3}-q_{0} q_{1}\right) \\
2\left(q_{1} q_{3}-q_{0} q_{2}\right) & 2\left(q_{2} q_{3}+q_{0} q_{1}\right) & \left(q_{0}^{2}-q_{1}^{2}-q_{2}^{2}+q_{3}^{2}\right)
\end{array}\right]
$$

After the transformation for vector $\underline{r}$ the following applies:

$$
\begin{aligned}
& \text { a) } \underline{r}=\underline{b}-\underline{p} \\
& \text { b) } \underline{\hat{r}}=\frac{\underline{b}-\underline{p}}{|\underline{b}-\underline{p}|} \text {, the unit vector representing the direction. } \\
& \text { c) } \underline{\rho}=\mu \underline{\hat{r}} \text {, the range }(\mu \text { is an unknown parameter }) \text {. }
\end{aligned}
$$

The range $\underline{\rho}$ represents the difference between the map point vector $\underline{m}$ and the position vector $\underline{p}$ and may be written as

$$
\underline{\rho}=\underline{m}-\underline{p} .
$$

The unit vector in the direction $\rho$ notated as $\underline{\hat{r}}$ in the navigation frame also may be evaluated as

$$
\hat{r}=\frac{\rho}{|\underline{\rho}|} .
$$

The quaternion vector $q=\left[\begin{array}{llll}q_{0} & q_{1} & q_{2} & q_{3}\end{array}\right]^{T}$ used in the transformation matrix (3) is with unity norm i.e.

$$
|\underline{q}|=\sqrt{q_{0}^{2}+q_{1}^{2}+q_{2}^{2}+q_{3}^{2}}=1 .
$$


This nice property of the quaternions can be used for transformation of the unit vector $\underline{\hat{r}}_{c}$ in the camera frame to the unit vector $\underline{\hat{r}}$ in the navigation frame:

$$
\underline{\hat{r}}=C_{b 2 n} \underline{\hat{r}}_{c} .
$$

We assume that we have information about the map point vector $\underline{m}$, the position vector from where the measurement was taken $p$ and single monocular camera mounted on the vehicle as described. We take observations with the camera of the map point and we want to determine the attitude of the vehicle, see figure 1 . Actually we want to compute the quaternion vector $q=\left[\begin{array}{llll}q_{0} & q_{1} & q_{2} & q_{3}\end{array}\right]^{T}$. The camera measurements give the unit vector $\underline{\hat{r}}_{c}$ in the camera frame (body frame) computed from equation (1). Using the map point vector $\underline{m}$ and the position vector $p$ we compute the range $\rho$ with equation (4). Next we use the range $\rho$ in equation (5) to calculate the unit vector $\underline{\hat{r}}$ in the navigation frame (replica of the single camera vector measurement).

Now that we have the two unit vectors, one in the camera frame (body frame) the other in the navigation frame, we can determine the quaternion vector $\underline{q}$.

Equation (7) written in matrix form is

$$
\left[\begin{array}{l}
\hat{r}_{x} \\
\hat{r}_{y} \\
\hat{r}_{z}
\end{array}\right]=\left[\begin{array}{ccc}
\left(q_{0}^{2}+q_{1}^{2}-q_{2}^{2}-q_{3}^{2}\right) & 2\left(q_{1} q_{2}-q_{0} q_{3}\right) & 2\left(q_{1} q_{3}+q_{0} q_{2}\right) \\
2\left(q_{1} q_{2}+q_{0} q_{3}\right) & \left(q_{0}^{2}-q_{1}^{2}+q_{2}^{2}-q_{3}^{2}\right) & 2\left(q_{2} q_{3}-q_{0} q_{1}\right) \\
2\left(q_{1} q_{3}-q_{0} q_{2}\right) & 2\left(q_{2} q_{3}+q_{0} q_{1}\right) & \left(q_{0}^{2}-q_{1}^{2}-q_{2}^{2}+q_{3}^{2}\right)
\end{array}\right]\left[\begin{array}{c}
\hat{r}_{c x} \\
\hat{r}_{c y} \\
\hat{r}_{c z}
\end{array}\right]
$$

\section{THE GAUSS-NEWTON METHOD}

Since the quaternion vector $\underline{q}$ is four elements vector additional equation should be added to equation (8) in order to solve the four unknowns in $\underline{q}$. The property of the quaternion vector to be with unity norm, equation (6) can be added. Equation (8) expressed in component form including equation (6) is:

$$
\begin{aligned}
& \hat{r}_{x}=\left(q_{0}^{2}+q_{1}^{2}-q_{2}^{2}-q_{3}^{2}\right) \hat{r}_{c x}+2\left(q_{1} q_{2}-q_{0} q_{3}\right) \hat{r}_{c y}+2\left(q_{1} q_{3}+q_{0} q_{2}\right) \hat{r}_{c z} \\
& \hat{r}_{y}=2\left(q_{1} q_{2}+q_{0} q_{3}\right) \hat{r}_{c x}+\left(q_{0}^{2}-q_{1}^{2}+q_{2}^{2}-q_{3}^{2}\right) \hat{r}_{c y}+2\left(q_{2} q_{3}-q_{0} q_{1}\right) \hat{r}_{c z} \\
& \hat{r}_{z}=2\left(q_{1} q_{3}-q_{0} q_{2}\right) \hat{r}_{c x}+2\left(q_{2} q_{3}+q_{0} q_{1}\right) \hat{r}_{c y}+\left(q_{0}^{2}-q_{1}^{2}-q_{2}^{2}+q_{3}^{2}\right) \hat{r}_{c z} \\
& 1=\sqrt{q_{0}^{2}+q_{1}^{2}+q_{2}^{2}+q_{3}^{2}}
\end{aligned}
$$

The nonlinear system of equations (9) can be written:

$$
Y_{n}=f\left(x_{n}, \theta\right)+Z_{n}
$$

This nonlinear system of equations includes four nonlinear equations and involves finding a solution, the quaternion vector $q$ such that every equation in the nonlinear system is 0. Efficient computing methods such as the Gauss-Newton method can be used for solving this problem.

An approach suggested by Gauss is to use a linear approximation to the nonlinear function to iteratively improve an initial guess $\theta^{0}$ for $\theta$ and keep improving the estimates until there is no change [5]. That is, we expand the nonlinear function $f\left(x_{n}, \theta\right)$ in a first order Taylor series about $\theta^{0}$ as

$$
f\left(x_{n}, \theta\right)=f\left(x_{n}, \theta^{0}\right)+v_{n 1}\left(\theta_{1}-\theta_{1}^{0}\right)+v_{n 2}\left(\theta_{2}-\theta_{2}^{0}\right)+\cdots+v_{n n}\left(\theta_{n}-\theta_{n}^{0}\right)
$$

where

$$
v_{n n}=\left.\frac{\partial f\left(x_{n}, \theta\right)}{\partial \theta_{n}}\right|_{\theta^{0}} \quad n=1,2, \ldots, N
$$

Incorporating all $N$ cases, we write

$$
\eta(\theta) \approx \eta\left(\theta^{0}\right)+V^{0}\left(\theta-\theta^{0}\right)
$$

where $V^{0}$ is the $N \times N$ derivative matrix with elements $\left\{v_{n n}\right\}$. This is equivalent to approximating the residuals, $z(\theta)=y-\eta(\theta)$, by

$$
z(\theta)=y-\left[\eta\left(\theta^{0}\right)+V^{0} \delta\right]=z^{0}-V^{0} \delta
$$

where $z^{0}=y-\eta\left(\theta^{0}\right)$ and $\delta=\theta-\theta^{0}$.

We then calculate the Gauss increment $\delta^{0}$ to minimize the approximate residual sum of squares $\left|z^{0}-V^{0} \delta\right|^{2}$, using

$$
\begin{gathered}
V^{0}=Q R=Q_{1} R_{1} \\
w_{1}=Q_{1}^{T} z^{0} \\
\hat{\eta}^{1}=Q_{1} w_{1}
\end{gathered}
$$

and so

$$
R_{1} \delta^{0}=w_{1}
$$

The point

$$
\hat{\eta}^{1}=\eta\left(\theta^{1}\right)=\eta\left(\theta^{0}+\delta^{0}\right)
$$

should now be closer to $y$ than $\eta\left(\theta^{0}\right)$, and so we move to this better parameter value $\theta^{1}=\theta^{0}+\delta^{0}$ and perform another iteration by calculating new residuals $z^{1}=y-\eta\left(\theta^{1}\right)$, a new derivative matrix $V^{1}$, and a new increment. This process is repeated until convergence is obtained, that is, until the increment is so small that there is no change in the elements of the parameter vector [5]. 
Convergence implies that the best estimates of the parameters are obtained. The Gauss-Newton method provides solution even if the system may not have a zero i.e. it returns a point where the residual is small. If the Jacobian of the system is singular the Gauss-Newton method might converge to a point that is not a solution of the system of equations.

\section{THE DAVENPORT Q-METHOD}

Spacecraft attitude determination is perhaps the oldest application and the attitude determination problem for a spacecraft from vector measurements was first formulated in [6]. Wahba formulated the attitude determination problem using vector observations as follows:

Given two sets of $n$ points $\left\{v_{1}, v_{2}, \ldots, v_{n}\right\}$, and $\left\{w_{1}, w_{2}, \ldots, w_{n}\right\}$, where $n \geq 2$, find the rotation matrix $R$ (i.e., the orthogonal matrix with determinant +1 ) which brings the first set into the best least squares coincidence with the second. That is find $R$ which minimizes

$$
\sum_{i=1}^{n}\left\|w_{i}-R v_{i}\right\|^{2}
$$

The matrix $R$ is a least squares estimate of the rotation matrix. In our case the vector $v_{i}$ is the unit vector $\underline{\hat{r}}_{c}$ in the camera frame i.e. body frame and the vector $w_{i}$ is the unit vector $\underline{\hat{r}}$ in the navigation frame (replica of the single camera vector measurement), see equation (8).

The elegant q-method as solution to the Wahba's problem was developed by Davenport [7]. The rational of the q-method is based on the following identity:

$$
\frac{1}{2} \sum_{i=1}^{n} a_{i}\left\|w_{i}-R v_{i}\right\|^{2}=1-q^{T} K q
$$

where the matrix $K$ is a function of the vector measurements.

The corresponding weights denoted by $a_{i}$, assume that they add to one i.e. $\sum_{i=1}^{n} a_{i}=1$, then the matrices $B$ and $S$, the column matrix $z$ and the scalar $\sigma$ are defined as follows:

$$
\begin{gathered}
B=\sum_{i=1}^{n} a_{i}\left(v_{i} w_{i}^{T}\right) \quad S=B+B^{T} \\
z=\left[B_{23}-B_{32} \quad B_{31}-B_{13} \quad B_{12}-B_{21}\right]^{T} \\
\sigma=\operatorname{tr}(B)
\end{gathered}
$$

where $\operatorname{tr}(\cdot)$ denotes the trace operator.
Next the $4 \times 4$ symmetric matrix $K$ of equation (18) is computed

$$
K=\left[\begin{array}{cc}
S-\sigma I & z \\
z & \sigma
\end{array}\right]
$$

Davenport showed that the optimal quaternion estimate $\underline{q}=\left[\begin{array}{llll}q_{0} & q_{1} & q_{2} & q_{3}\end{array}\right]^{T}$ is an eigenvector of the matrix $K$. However there are four eigenvalues and they each have different eigenvectors. The eigenvector which corresponds to the largest eigenvalue of the matrix $K$ is the least squares optimal estimate of the attitude.

\section{SimUlation RESUlts}

In the simulation we assumed that Inertial Measurement Unit (IMU) is mounted at the center of gravity of the vehicle. The measurements (angular rates) from the gyros are used to determine the attitude of the vehicle, the quaternion vector $\underline{q}=\left[\begin{array}{llll}q_{0} & q_{1} & q_{2} & q_{3}\end{array}\right]^{T}$. The instrumentation, computation, alignment, environment errors make the gyro measurements diverge slowly from the real measurements with time, so as the vehicle attitude.

When the Gauss-Newton method was tested the vehicle flies on a circular path around four map points, see figure 2 . Positions of the map points are known $M_{1}=\left[\begin{array}{llll}370 & 500 & 3\end{array}\right]^{T}, M_{2}=\left[\begin{array}{llll}400 & 550 & 2\end{array}\right]^{T}$, $M_{3}=\left[\begin{array}{lll}430 & 500 & 3\end{array}\right]^{T}$ and $M_{4}=\left[\begin{array}{lll}400 & 480 & 2\end{array}\right]^{T}$. The monocular camera has focal length of $8 \mathrm{~mm}(f=0.008 \mathrm{~m})$. The observation errors (camera measurements) have variance of $5 \mathrm{~nm}\left(\sigma^{2}=5 \cdot 10^{-9} \mathrm{~m}\right)$.

From figure 3 to figure 6 the errors in the quaternion elements of the quaternion vector $\underline{q}=\left[\begin{array}{llll}q_{0} & q_{1} & q_{2} & q_{3}\end{array}\right]^{T}$ which represents the vehicle attitude are shown. We can see the divergent (black line) and the estimated (gray line) errors.

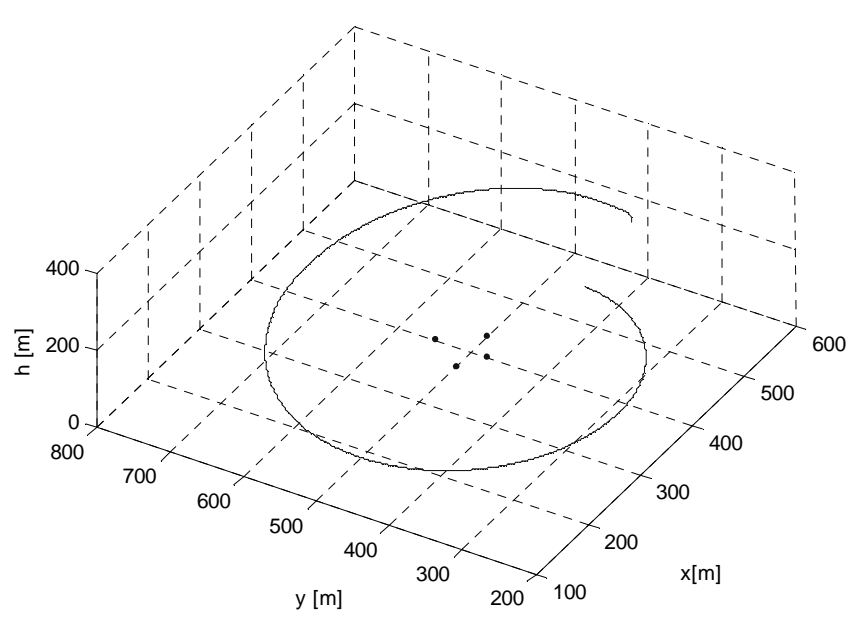

Figure 2. Vehicle trajectory around four known map points 
When we used four known map points the problem of attitude determination becomes overdetermined, thus equation (9) becomes overdetermined nonlinear system of equations. The Gauss-Newton method was tested with one map point estimate but the simulation results turn not to improve much.

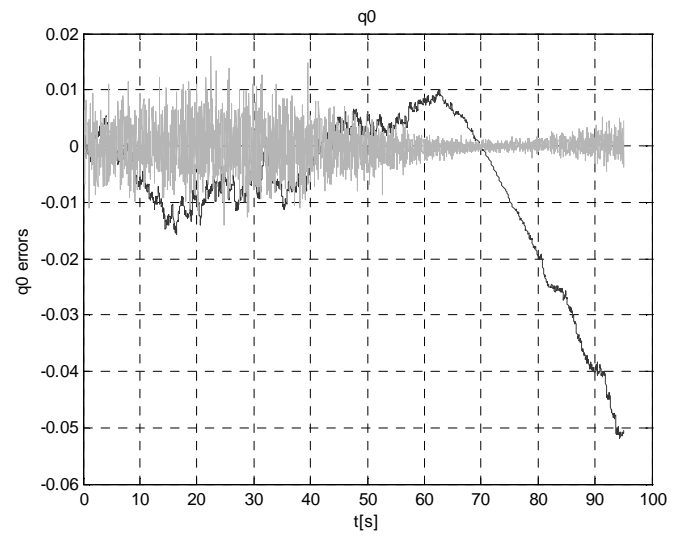

Figure 3. $q_{0}$ element errors

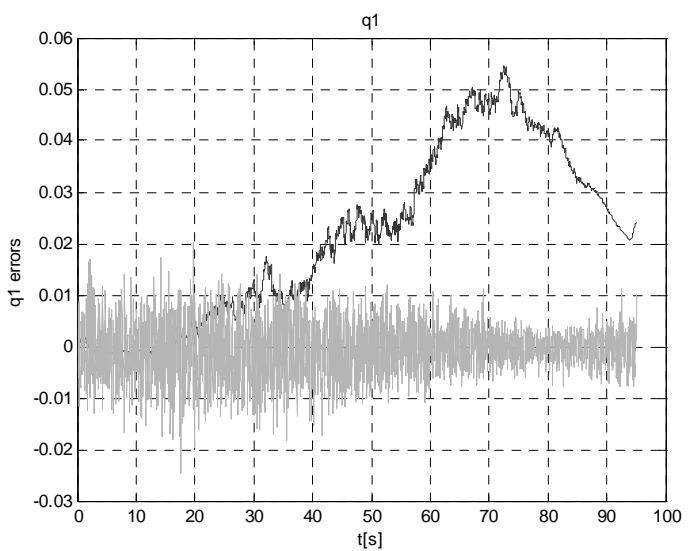

Figure 4. $q_{1}$ element errors

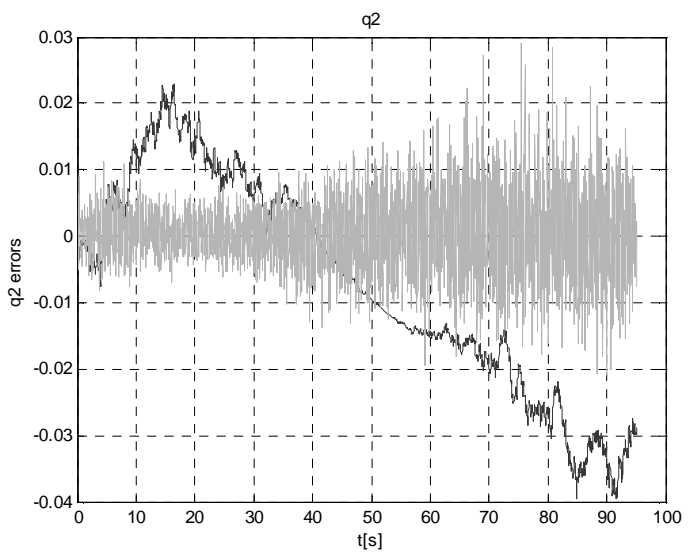

Figure 5. $q_{2}$ element errors
IEEE Intelligent Systems Conference, London, July 2010

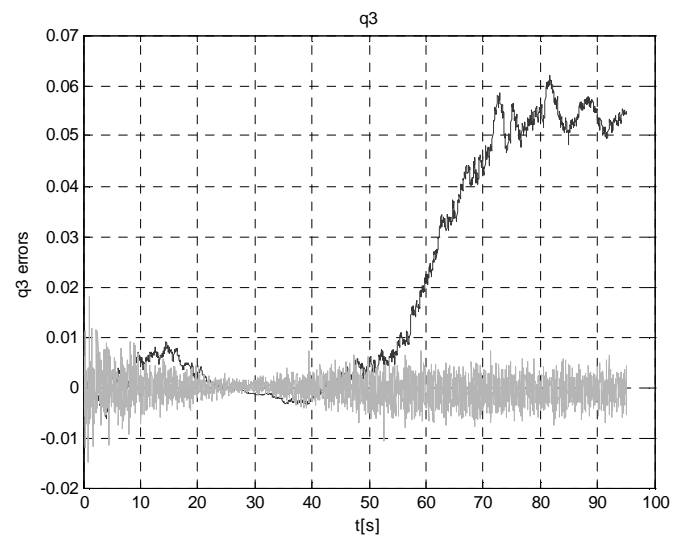

Figure 6. $q_{3}$ element errors

As this method is very sensitive to the initial guess (here we have used the divergent attitude determined from the IMU) using two or more map points are must in order to obtain satisfactory results, as shown on figure 3 to figure 6 .

The camera vector observations are always corrupted by random errors which may cause divergence of the GaussNewton method. In our case we used observation errors with variance of $5 \mathrm{~nm}\left(\sigma^{2}=5 \cdot 10^{-9} \mathrm{~m}\right)$.

In the simulation of the Davenport q-method the vehicle flies on a circular path around same four map points, see figure 7. The positions of the map points are known. The monocular camera has the same specifications. From figure 8 to figure 11 the errors in the quaternion elements of the quaternion vector $\underline{q}=\left[\begin{array}{llll}q_{0} & q_{1} & q_{2} & q_{3}\end{array}\right]^{T}$ are shown. We can see the divergent (black line) and the estimated (gray line) errors. As with the Gauss-Newton method using two or more map points are must in order to obtain satisfactory results, as shown on figure 8 to figure 11 .

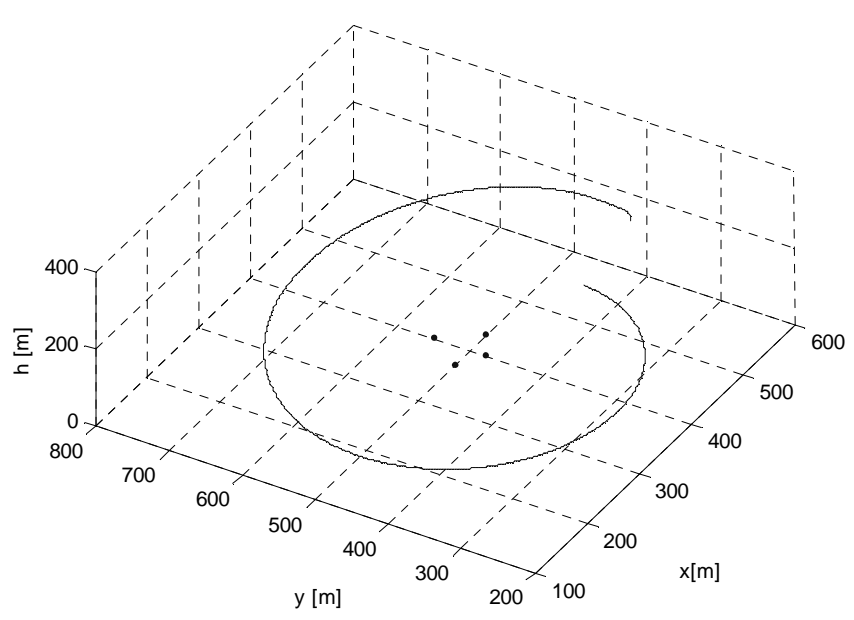

Figure 7. Vehicle trajectory around four known map points 
Davenport q-method has considerable advantages over the Gauss-Newton method in that it does not require initial guess of the attitude, it does not require linearization of some equation model, the covariance of the estimate can be calculated and it may be extended to a case where the measurements have different individual variances.

The notes on the Davenport q-method are as follows. Since it requires eigenproblem to be solved the solution is numerically "intensive".

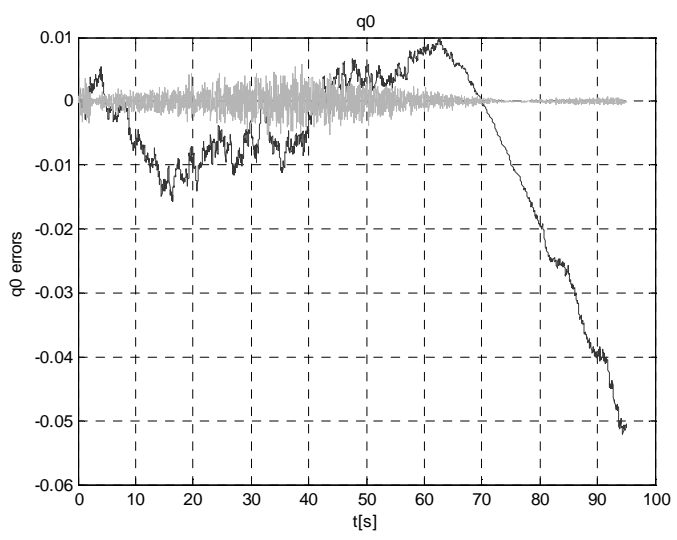

Figure 8. $q_{0}$ element errors

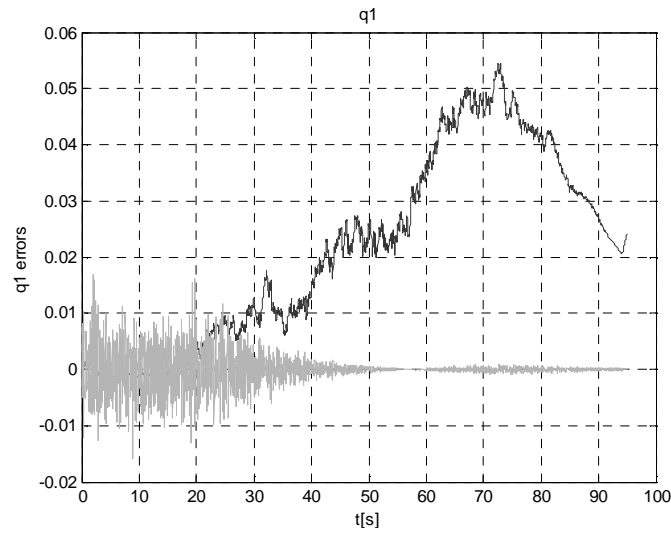

Figure 9. $q_{1}$ element errors

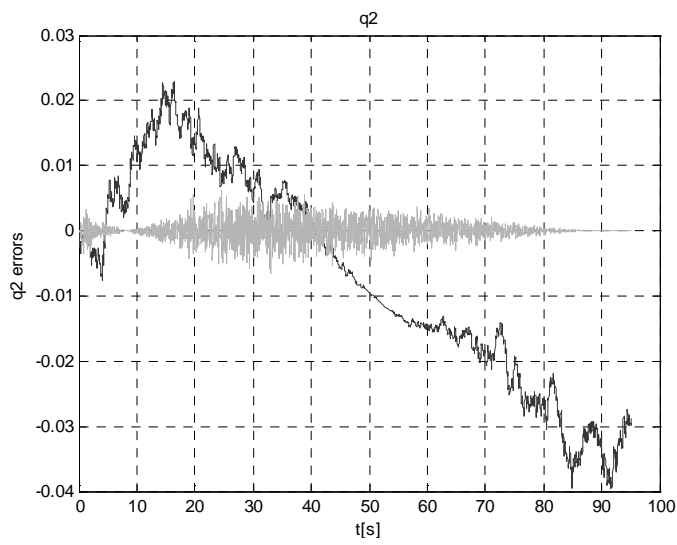

Figure 10. $q_{2}$ element errors
IEEE Intelligent Systems Conference, London, July 2010

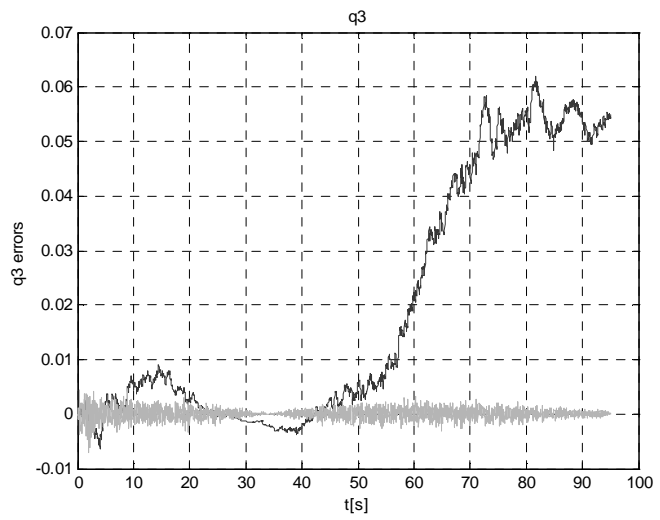

Figure 11. $q_{3}$ element errors

The case when two largest eigenvalues of $K$ are equal is not a failure of the q-method; it means that the data aren't sufficient to determine the attitude uniquely [8].

\section{CONCLUSIONS AND FUTURE WORK}

Attitude determination in many means is a complicated problem. In this paper we have presented a proper framework for attitude determination from single camera vector observations in a known environment. The Gauss Newton method and the Davenport q-method were put on the test. The simulation results show that the Davenport q-method is a preferred choice. Based on the Davenport q-method many algorithms such as the QUEST algorithm [9] provide less "intensive" way to estimate the solution to the eigenproblem and will be included in the future research. Also in our future work we look for a way how to determine attitude from single camera vector observations when the environment is unknown. The SLAM theory in particular BOSLAM has taken our attention.

\section{REFERENCES}

[1] D.H.Shim, H.Chung, H.J.Kim and S.Sastry "Autonomous Exploration in Unknown Urban Environments for Unmanned Aerial Vehicles" AIAA Guidance Navigation and Control Conference, 2005.

[2] R. Sivakumar and R. Sengupta. "Safe UAV Navigation with Sensor Processing Delays in an Unknown Environment", 43rd IEEE Conference on Decision and Control, p.1081-1086, 2004.

[3] H.Goldstein. "Classical Mechanics", Second edition, Addison-Wesley, Boston, MA, 1980.

[4] J.A. Farrell and M. Barth.’The Global Positioning System and Inertial Navigation", The McGraw-Hill Inc., 1999.

[5] D.M.Bates and D.G.Watts "Nonlinear Regresion Analysis and its Applications", John Wiley and Sons Inc., 1988.

[6] G.Wahba, "A Least Squares Estimate of Spacecraft Attitude", SIAM Review, Vol. 7, No. 3, 1965.

[7] P. D. Davenport, "A Vector Approach to the Algebra of Rotations with Applications", Goddard Space Flight Center, November, 1968.

[8] F. L. Markley, "Fast Quatenion Attitude Estimation from Two Vector Measurements" NASA Goddard Space Flight Center.

[9] M. D. Shuster and S. D. Oh, "Three-axis attitude determination from vector observations" J. Guid. Control, vol. 4, no. 1, pp. 70-77, 1981. 